Physics

Physics Research Publications

Purdue University

Year 2005

\begin{abstract}
A direct method for locating minimum-energy crossing points (MECPs) in spin-forbidden transitions and nonadiabatic reactions
\end{abstract}

T. Chachiyo J. H. Rodriguez

This paper is posted at Purdue e-Pubs.

http://docs.lib.purdue.edu/physics_articles/127 


\title{
A direct method for locating minimum-energy crossing points (MECPs) in spin-forbidden transitions and nonadiabatic reactions
}

\author{
Teepanis Chachiyo and Jorge $\mathrm{H}$. Rodriguez ${ }^{\mathrm{a})}$ \\ Department of Physics, Purdue University, West Lafayette, Indiana 47907-2036
}

(Received 25 February 2005; accepted 30 June 2005; published online 8 September 2005)

\begin{abstract}
An efficient computational method for locating minimum-energy crossing points (MECPs) between potential-energy surfaces in spin-crossover transitions and nonadiabatic spin-forbidden (bio)chemical reactions is introduced. The method has been tested on the phenyl cation and the computed MECP associated with its radiationless singlet-triplet spin crossover is in good agreement with available data. However, the convergence behavior of the present method is significantly more efficient than some alternative methods which allows us to study nonadiabatic processes in larger systems such as spin crossover in metal-containing compounds. The convergence rate of the method obeys a fast logarithmic law which has been verified on the phenyl cation. As an application of this new methodology, the MECPs of the ferrous complex $\left[\mathrm{Fe}(\mathrm{ptz})_{6}\right]\left(\mathrm{BF}_{4}\right)_{2}$, which exhibits light-induced excited spin state trapping, have been computed to identify their geometric and energetic parameters during spin crossover. Our calculations, in conjunction with spin-unrestricted density-functional calculations, show that the transition from the singlet ground state to a triplet intermediate and to the quintet metastable state of $\left[\mathrm{Fe}(\mathrm{ptz})_{6}\right]\left(\mathrm{BF}_{4}\right)_{2}$ is accompanied by unusually large bond-length elongations of the axial ligands $(\approx 0.26$ and $0.23 \AA$, respectively). Our results are consistent with crystallographic data available for the metastable quintet but also predict new structural and energetic information about the triplet intermediate and at the MECPs which is currently not available from experiment. (C) 2005 American Institute of Physics.

[DOI: $10.1063 / 1.2007708]$
\end{abstract}

\section{INTRODUCTION}

Many processes, such as radiationless spin crossover in coordination compounds, involve transitions between multielectronic states of different spin multiplicity. Such transitions, not being electric dipole allowed, are formally spin forbidden and are often interpreted in terms of the crossing of two energy hypersurfaces. Likewise, many (bio)chemical reactions, such as dioxygen binding in iron heme proteins, are particular types of nonadiabatic processes in which there is a net change in the system's total electronic spin. As such these reactions are called spin forbidden. Energy surface crossings play important roles in understanding the mechanisms and dynamics of many nonadiabatic chemical and biochemical processes ${ }^{1-4}$ and, in particular, when a process involves a change in the total electronic spin. Determining the lowest crossing point, at which two energy surfaces corresponding to different spin states intersect with minimum energy, is important because it serves as the most likely place where the transition occurs. ${ }^{1,5,6}$

To elucidate the mechanisms associated with spinforbidden processes one can use a simple and yet powerful concept, namely, the minimum-energy crossing point (MECP) on the seam line at which two adiabatic surfaces intersect (Fig. 1). For an $N$-atom molecular system, the MECP corresponds to a set of $3 N$ nuclear coordinates, $\left\{\mathbf{R}_{\mathbf{i}}\right\}$, for which the energies of two spin states are equal and often

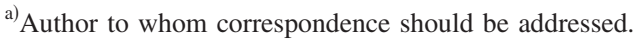

represents a key bottleneck along the pathway of spinforbidden processes. ${ }^{5,6}$ Therefore, it is important to compute MECPs in order to understand and quantify the physical interactions which are active at the crossing point, such as spin-orbit coupling (SOC), which facilitate or "catalyze" spin-forbidden processes. Although in principle existing electronic structure methods can calculate independently two different adiabatic surfaces and indirectly find their crossing points by superposition, this is in practice impossible for large transition metal-containing complexes such as coordination compounds and metal centers in proteins. Therefore, it is crucial to develop algorithms for finding MECPs without having to compute entire potential-energy surfaces since this is, in general, prohibitive. However, most electronic structure packages presently compute with good efficiency stationary points on single potential-energy surfaces (e.g., energy minima, saddle points) but typically do not compute MECPs because these are not stationary points on either of the two intersecting spin surfaces. ${ }^{3}$ This is particularly true for computations on large metal-containing systems. Here, we report the computational implementation of a method for locating MECPs on the seam of two crossing energy surfaces. This is carried out in a fairly direct fashion by using efficient energy-gradient methods in conjunction with densityfunctional calculations.

Previous investigators have made significant advances towards the direct calculation of MECPs..$^{1,7,8}$ However, these methods have been mostly applied to small organic systems and are not practical for the evaluation of MECPs of large 


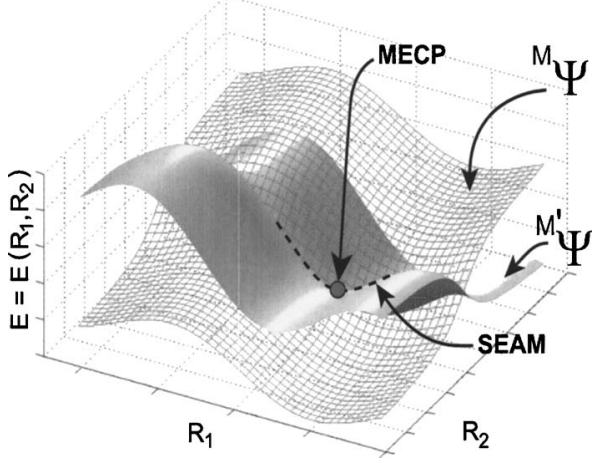

FIG. 1. Potential-energy surfaces corresponding to two multielectronic states of different spin multiplicities ${ }^{M} \Psi$, and ${ }^{M^{\prime}} \Psi$, as a function of two nuclear coordinates $R_{1}$ and $R_{2}$. The lowest-energy point along the line of intersection (seam) is the minimum-energy crossing point (MECP).

metal complexes when used in conjunction with expensive correlated wave-function-based methods. In particular, some constrained algorithms for the investigation of nonadiabatic surface crossing use analytic energy gradients with constraints enforced by the method of Lagrange multipliers ${ }^{5-9}$ and also computationally expensive multiconfiguration selfconsistent-field (MCSCF) configuration-interaction (CI) wave functions. More recently, however, density-functional methods have also been used. ${ }^{10,11}$ These algorithms do allow the determination of energetically accessible portions of crossing hypersurfaces without having to characterize the entire crossing surfaces but also have practical limitations. In particular, these impose arbitrary equality constraints on some bond lengths and angles ${ }^{6}$ and use MCSCF/CI wave functions which are not only impractical for large metal complexes but also only in part recover dynamic correlation energy. ${ }^{12}$ This latter fact in turn affects the accuracy of the location of crossing points between surfaces which have different intrinsic measures of correlation energy, as has been documented for the phenyl cation. ${ }^{12}$ Consequently, to treat metal-containing systems we need to develop fastconverging MECP algorithms which are based on less expensive $a b$ initio methods, such as density-functional theory, and which also recover to a greater extent dynamic correlation effects. Some methods ${ }^{1,12}$ are an initial step in this direction and have been the starting point of our own development.

One main problem with some pioneering algorithms has been their poor convergence behavior which shows problematic oscillations that increase the computational cost. ${ }^{1,12}$ In this work, we present a new method of locating crossing points between nonadiabatic potential-energy surfaces that exhibits a fast, nonoscillating, convergence rate and which is suitable for the study of nonadiabatic processes in large molecular systems. For example, the method can be applied to study spin crossover in metal coordination compounds and also spin-forbidden ligand-binding reactions in metalloproteins. In this paper we first detail a new MECP methodology which has been computationally implemented. Second, the convergence behavior of the new method is analyzed in detail. Third, test calculations are presented for a simple representative system, namely, the phenyl cation, to compare our results with previously published work. Finally, the algorithm is used to study the spin-crossover pathway of $\left[\mathrm{Fe}(\mathrm{ptz})_{6}\right]\left(\mathrm{BF}_{4}\right)_{2} \quad(\mathrm{ptz}=1$-propyltetrazole $),{ }^{13}$ a remarkable complex which exhibits light-induced excited spin state trapping $^{14,15}$ (LIESST) and which is potentially useful in molecular-level memory storage and display technologies. ${ }^{15,16}$

\section{THEORETICAL METHODS}

\section{A. MECP methodology}

We consider a molecular system containing $N$ atoms whose electronic wave functions ${ }^{M} \Psi$ and ${ }^{M^{\prime}} \Psi$ correspond to states of different spin multiplicity, $M=2 S+1$ and $M^{\prime}=2 S^{\prime}$ +1 , respectively. For each state the total electronic energy within the Born-Oppenheimer approximation is a function of $3 N$ nuclear coordinates,

$$
\begin{aligned}
& E^{M} \equiv E^{M}\left(R_{1}, R_{2}, \ldots, R_{3 N}\right)=E^{M}\left(\mathbf{R}_{\mathbf{i}}\right), \\
& E^{M^{\prime}} \equiv E^{M^{\prime}}\left(R_{1}, R_{2}, \ldots, R_{3 N}\right)=E^{M^{\prime}}\left(\mathbf{R}_{\mathbf{i}}\right),
\end{aligned}
$$

where a particular set of coordinates, $\mathbf{i}=\left\{R_{1}, R_{2}, \ldots, R_{3 N}\right\}$, defines a $3 N$-dimensional vector $\mathbf{R}_{\mathrm{i}}=R_{1} \hat{\epsilon}_{1}+R_{2} \hat{\epsilon}_{2} \ldots+R_{3 N} \hat{\epsilon}_{3 N}$. Thus, for each state ${ }^{M} \Psi$ the energy landscape of a molecular system is $3 N$ dimensional. By varying $\mathbf{R}_{\mathbf{i}}$ one can construct hyperdimensional energy surfaces as illustrated by Fig. 1 which also depicts the seam of intersection with its global minimum (i.e., MECP).

Some methods for locating lowest-energy crossing points explicitly minimize $E^{M}\left(\mathbf{R}_{\mathbf{i}}\right)$ but only use $E^{M^{\prime}}\left(\mathbf{R}_{\mathbf{i}}\right)$ to direct the computational search towards the seam. ${ }^{1,12}$ However, our results in Sec. III show that treating both energy surfaces on an equal footing speeds up the convergence and significantly lowers the computational cost of the calculations. Accordingly, the implementation of our MECP algorithm explicitly considers both energy surfaces, $E^{M}$ and $E^{M^{\prime}}$, by defining an energy function $U\left(\mathbf{R}_{\mathbf{i}}\right)$ to be minimized subject to a constraint $C$,

$$
\begin{aligned}
& U\left(\mathbf{R}_{\mathbf{i}}\right)=\left(E^{M}+E^{M^{\prime}}\right) / 2, \\
& C\left(\mathbf{R}_{\mathbf{i}}\right)=\left(E^{M}-E^{M^{\prime}}\right)^{n}=0,
\end{aligned}
$$

where, for example, $n=1,2,3$. In our approach finding a MECP is equivalent to minimizing $U\left(\mathbf{R}_{\mathbf{i}}\right)$, subject to condition $C$, where $E^{M}$ and $E^{M^{\prime}}$ are evaluated from self-consistent spin density-functional theory, ${ }^{17-19}$ (SDFT) calculations at each $\mathbf{R}_{\mathbf{i}}$.

Trying to minimize both energy surfaces simultaneously, via Eq. (3), may initially seem unnecessary because Eq. (4) guarantees that $E^{M}$ and $E^{M^{\prime}}$ become equal at the MECP. However, as illustrated by Figs. 2 and 3, this procedure significantly reduces the CPU time, relative to an alternative method, in two ways: (i) the number of iteration steps needed to reach the MECP is significantly reduced and (ii) the number of self-consistent-field (SCF) cycles used in SDFT evaluations of $E^{M}\left(\mathbf{R}_{i}\right)$ and $E^{M^{\prime}}\left(\mathbf{R}_{\mathbf{i}}\right)$ at each iteration is generally lower since SCF wave functions of previous iterations are better initial guesses for subsequent iterations. 


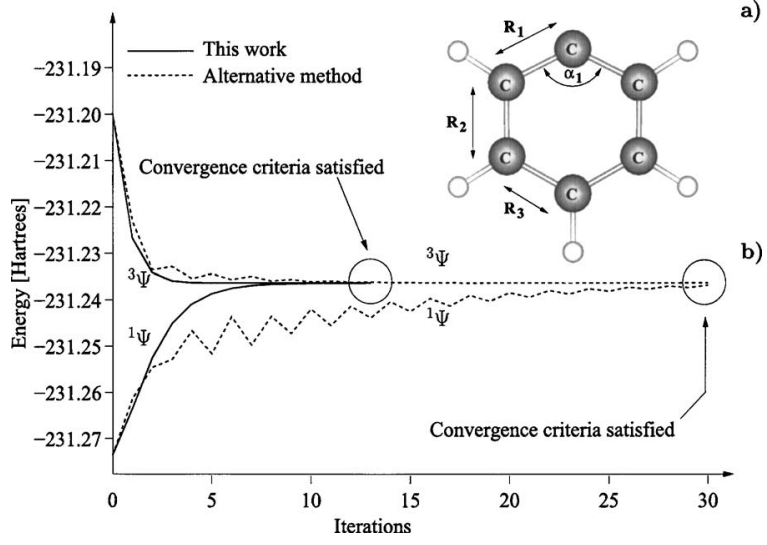

FIG. 2. (a) Optimized geometry of phenyl cation $\left[\mathrm{C}_{6} \mathrm{H}_{5}\right]^{+}$with parameters given in Table I. (b) Convergence of energy surfaces toward the MECP by using our fast-converging energy-gradient algorithm (solid lines) and by our implementation of alternative (Refs. 12 and 35) methods (dotted lines). The number of iterations $(\approx 13)$ needed to find the $\mathrm{MECP}^{3 \leftrightarrow 1}$ with our algorithm is significantly lower than the number needed $(\approx 31)$ for the alternative method. As shown in Table II, we located the MECP $20.73 \mathrm{kcal} / \mathrm{mol}$ above the equilibrium geometry energy in close agreement with other significantly more expensive methods (Refs. 1, 12, and 35).

Our MECP algorithm is based on the Newton-Raphson method which is an efficient minimization procedure. ${ }^{20}$ To minimize a function $U\left(\mathbf{R}_{\mathbf{i}}\right)$ subject to a condition $C=0$, [Eqs. (3) and (4)] one can evaluate a gradient vector $\mathbf{g}$, a Jacobi vector $\mathbf{J}$, and a Hessian matrix $\widetilde{H}$ whose elements are given by $^{20}$

$$
\begin{aligned}
& g_{i}=\partial U / \partial R_{i}, \\
& J_{i}=\partial C / \partial R_{i}, \\
& H_{i j}=\partial^{2} U / \partial R_{i} \partial R_{j},
\end{aligned}
$$

where $i=1,2, \ldots, 3 N$. Putting Eqs. (3) and (4) in the context of Eqs. (5)-(7) one obtains

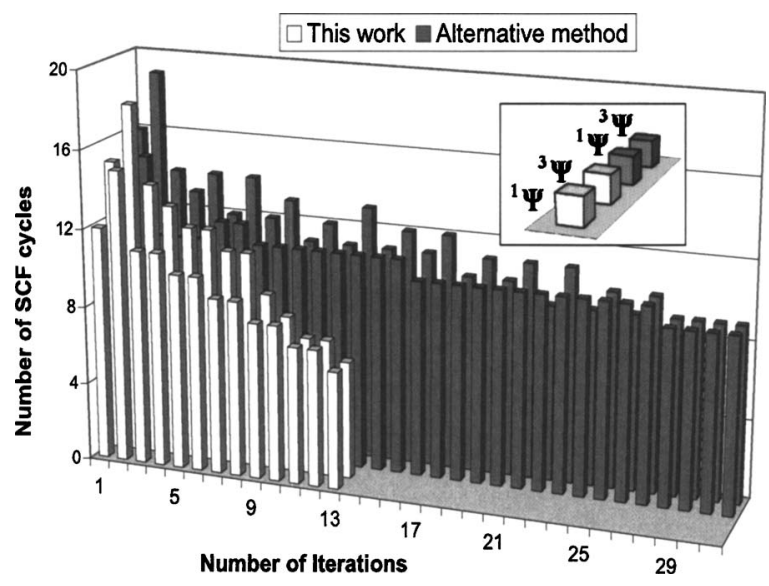

FIG. 3. MECP convergence histogram for phenyl cation. The energies $E^{1}\left(\mathbf{R}_{\mathbf{i}}\right)$ and $E^{3}\left(\mathbf{R}_{\mathbf{i}}\right)$ are computed at each iteration $\mathbf{i}$ from the SCF-SDFT calculations for each set of nuclear coordinates $\mathbf{R}_{\mathbf{i}}$. The figure shows that (i) the number of SCF cycles needed to converge to each SDFT energy, $E^{1}\left(\mathbf{R}_{\mathbf{i}}\right)$ and $E^{3}\left(\mathbf{R}_{\mathrm{i}}\right)$, with our MECP algorithm is always lower than the number needed for our implementation of an alternative method (Refs. 1 and 12) and (ii) the number of iterations needed to find $\mathrm{MECP}^{3 \leftrightarrow 1}$ is also lower with our algorithm than the number needed for the alternative method.

$$
\begin{aligned}
& \mathbf{g}=\left(\mathbf{g}^{M}+\mathbf{g}^{M^{\prime}}\right) / 2 \\
& \mathbf{J}=2\left(E^{M}-E^{M^{\prime}}\right)\left(\mathbf{g}^{M}-\mathbf{g}^{M^{\prime}}\right), \\
& \widetilde{\mathbf{H}}=\left(\widetilde{H}^{M}+\widetilde{H}^{M^{\prime}}\right) / 2,
\end{aligned}
$$

where $E^{M}, E^{M^{\prime}}, \mathbf{g}^{M}, \mathbf{g}^{M^{\prime}}, \widetilde{H}^{M}, \widetilde{H}^{M^{\prime}}$ are the energy, gradient, and Hessian of each energy surface evaluated at iteration $\mathbf{i}$ (i.e., at current set of nuclear coordinates). In our work, these quantities are evaluated from the SDFT (Refs. 17 and 19) calculations at each iteration.

Depending on the size of the molecular system, it may not be practical to compute the Hessian matrices analytically. Instead, these may be evaluated numerically using the following recursive relations for each multielectronic state: $:^{21,22}$

$$
\widetilde{H}_{(i+1)}^{M^{\prime}}=\widetilde{H}_{(i)}^{M^{\prime}}+\frac{\left(d \mathbf{g}^{M^{\prime}}\right)\left(d \mathbf{g}^{M^{\prime}}\right)^{T}}{d \mathbf{g}^{M^{\prime}} \cdot d \mathbf{R}}+\frac{\widetilde{H}_{(i)}^{M^{\prime}}(d \mathbf{R})(d \mathbf{R})^{T} \widetilde{H}_{(i)}^{M^{\prime}}}{d \mathbf{R} \cdot \widetilde{H}_{(i)}^{M^{\prime}} d \mathbf{R}},
$$

$$
\begin{aligned}
& d \mathbf{g}^{M^{\prime}}=\mathbf{g}_{i+1}^{M^{\prime}}-\mathbf{g}_{i}^{M^{\prime}}, \\
& d \mathbf{R}=\mathbf{R}_{i+1}-\mathbf{R}_{i},
\end{aligned}
$$

where $d \mathbf{R}$ is a stepping vector. Therefore, as one takes a step on the energy surface, $\mathbf{R}_{i} \rightarrow \mathbf{R}_{i+1}$, the Hessian can be updated accordingly. Such updating scheme has been successfully applied to the calculation of equilibrium molecular structures within the Berny optimization method. ${ }^{22}$ As demonstrated in Sec. III, these recursive relations also produce good results in the calculation of MECPs.

During the iterative search for a MECP we define a vector $\mathbf{R}_{\mathbf{0}}$ corresponding to an initial guess of the nuclear coordinates and the stepping vector $d \mathbf{R}$. Then, a vector $\mathbf{R}_{\mathbf{1}}$ corresponding to a set of coordinates that, subject to condition $C$, brings $U\left(\mathbf{R}_{\mathbf{i}}\right)$ closer to its minimum can be evaluated as follows: $:^{20}$

$$
\begin{aligned}
& \mathbf{R}_{\mathbf{1}}=\mathbf{R}_{\mathbf{0}}+d \mathbf{R}, \\
& d \mathbf{R}=-\widetilde{H}^{-1}(\mathbf{g}+\lambda \mathbf{J}), \\
& \lambda=\frac{C-\mathbf{J} \cdot\left(\tilde{H}^{-1} \mathbf{g}\right)}{\mathbf{J} \cdot\left(\tilde{H}^{-1} \mathbf{J}\right)} .
\end{aligned}
$$

For molecular systems the previous expressions can be quite problematic because these contain three translational and three rotational degrees of freedom. ${ }^{23}$ As a consequence, the stepping vector $d \mathbf{R}$ may unnecessarily lead the search through translations or rotations that do not change the energy $U\left(\mathbf{R}_{\mathbf{i}}\right)$. To overcome these problems and to have more control over $d \mathbf{R}$, Eqs. (15) and (16) can be rewritten in terms of an eigenvalue problem,

$$
d \mathbf{R}=-\sum_{i} \frac{\left(\alpha_{i}+\lambda \beta_{i}\right)}{\epsilon_{i}} \mathbf{h}_{i},
$$


TABLE I. Geometric parameters of $\left[\mathrm{C}_{6} \mathrm{H}_{5}\right]^{+1} A_{1}$ ground state and MECP.

\begin{tabular}{llccccl}
\hline \hline & Method & $R_{1}$ & $R_{2}$ & $R_{3}$ & $\alpha_{1}$ & Reference \\
\hline${ }^{1} A_{1}$ minimum & UB3LYP/cc-pVTZ & 1.318 & 1.431 & 1.388 & 147.7 & This work \\
& B3LYP/SV & 1.339 & 1.447 & 1.405 & 144.5 & 12 \\
\multirow{4}{*}{ MECP } & B3LYP/6-31G(d) & 1.327 & 1.435 & 1.395 & 147.3 & 35 \\
& UB3LYP/cc-pVTZ & 1.400 & 1.372 & 1.425 & 128.9 & This work \\
& B3LYP/SV & 1.415 & 1.392 & 1.437 & 128.9 & 12 \\
\hline \hline
\end{tabular}

$$
\lambda=\left(C-\sum_{i} \frac{\alpha_{i} \beta_{i}}{\epsilon_{i}}\right) /\left(\sum_{i} \frac{\beta_{i} \beta_{i}}{\epsilon_{i}}\right),
$$

$$
\widetilde{H} \mathbf{h}_{i}=\epsilon_{i} \mathbf{h}_{i}
$$

$$
\alpha_{i}=\mathbf{g} \cdot \mathbf{h}_{i},
$$

$$
\beta_{i}=\mathbf{J} \cdot \mathbf{h}_{i},
$$

where $\epsilon_{i}$ and $\mathbf{h}_{i}$ are the eigenvalues and eigenvectors, respectively, of the Hessian matrix and the summation is over all nonzero $\epsilon_{i}$ to avoid unnecessary rotational and translational degress of freedom. ${ }^{23}$ The eigenvectors are the normal modes and the eigenvalues the corresponding vibrational frequencies. Supplementary Fig. 1 illustrates the overall algorithm. ${ }^{40}$

\section{B. An alternative MECP method}

A more conventional method for locating MECPs has been used in conical intersection analyses ${ }^{1}$ and is briefly summarized as follows. Within the vicinity of the seam line between two energy surfaces, a vector is defined which points directly to the seam

$$
\mathbf{f}=\left(E_{A}-E_{B}\right)\left(\mathbf{g}_{A}-\mathbf{g}_{B}\right) .
$$

It follows that a vector which is perpendicular to $\mathbf{f}$ would be tangent to the seam. Such tangential vector can be obtained by a Gram-Schmidt orthogonalization procedure. ${ }^{1}$ Traveling along the direction of these vectors is equivalent to moving along the seam line until reaching its minimum. Putting this method in the context of the Newton-Raphson procedure,

$$
\begin{aligned}
& \mathbf{g}=\left(E_{A}-E_{B}\right)\left(\mathbf{g}_{A}-\mathbf{g}_{B}\right)+\mathbf{g}_{A}-\frac{\mathbf{g}_{A}-\mathbf{g}_{B}}{\left|\mathbf{g}_{A}-\mathbf{g}_{B}\right|}\left[\mathbf{g}_{A} \cdot \frac{\mathbf{g}_{A}-\mathbf{g}_{B}}{\left|\mathbf{g}_{A}-\mathbf{g}_{B}\right|}\right], \\
& \widetilde{H}=\frac{\left|\mathbf{g}_{A}\right|}{\left|\mathbf{g}_{A}-\mathbf{g}_{B}\right|} \widetilde{H}_{B}-\frac{\left|\mathbf{g}_{B}\right|}{\left|\mathbf{g}_{A}-\mathbf{g}_{B}\right|} \widetilde{H}_{A}, \\
& \vec{J}=0
\end{aligned}
$$

$$
C=0 .
$$

One basic difference between this alternative method and the present work is how gradients and Hessians are defined. This can be appreciated by comparing Eqs. (23)-(26) with Eqs. (8)-(10). The results of calculations with our own method (vide supra) and with this alternative method are shown in Tables I and II and Figs. 2 and 3.

\section{Computation of relevant energies by spin density-functional theory}

For a molecular system composed of $N$ atoms, a particular set of nuclear coordinates, $\mathbf{i}=\left\{R_{1}, \ldots, R_{3 N}\right\}$, defines a $3 N$-dimensional vector $\mathbf{R}_{\mathrm{i}}=R_{1} \hat{\epsilon}_{1}+\cdots+R_{3 N} \hat{\epsilon}_{3 N}$ (vide supra). Within the Born-Oppenheimer approximation a multielectronic state ${ }^{M} \Psi\left(\mathbf{r}, \mathbf{R}_{\mathbf{i}}\right)$ depends explicitly on the electronic coordinates $\mathbf{r}$ but only parametrically on the nuclear coordinate vector $\mathbf{R}_{\mathbf{i}}{ }^{24}$ Thus, to compute potential-energy surfaces corresponding to a spin multiplicity $M$ we solve explicitly the electronic structure problem for a given $\mathbf{R}_{\mathbf{i}}$ and obtain electronic energies as a function of the nuclear coordinates $E^{M}\left(\mathbf{R}_{\mathbf{i}}\right)$.

The electronic energies required by the MECP algorithm are computed from spin density-functional theory ${ }^{17-19}$ (SDFT) calculations on the molecular geometries of interest (Supplementary Fig. 1). Density-functional methods can account for electron correlation effects at a computational cost that is much lower than some correlated wave-functionbased methods of comparable accuracy ${ }^{25,26}$ such as MøllerPlesset perturbation theory (MP2). In particular, the relatively recent development of gradient-corrected exchangecorrelation functionals ${ }^{27-32}$ makes of SDFT a well-suited method for treating large open-shell metal complexes, such as the one studied here, where correlation effects are important. ${ }^{33,34}$ Thus, SDFT calculations are ideally suited for evaluating the energy surfaces, $E^{M}\left(\mathbf{R}_{\mathbf{i}}\right)$ and $E^{M^{\prime}}\left(\mathbf{R}_{\mathbf{i}}\right)$, and accurate relative energies ${ }^{34}$ which are needed to compute MECPs of fairly large metal-containing complexes.

TABLE II. Energies of selected geometries of $\left[\mathrm{C}_{6} \mathrm{H}_{5}\right]^{+}$relative to ${ }^{1} A_{1}$ state.

\begin{tabular}{lcccc}
\hline \hline Method & $\begin{array}{c}E\left({ }^{1} A_{1} \text { min. }\right) \\
{[\text { hartree }]}\end{array}$ & $\begin{array}{c}E(\mathrm{MECP}) \\
{[\mathrm{kcal} / \mathrm{mol}]}\end{array}$ & $\begin{array}{c}E\left({ }^{3} B_{1} \mathrm{~min} .\right) \\
{[\mathrm{kcal} / \mathrm{mol}]}\end{array}$ & Reference \\
\hline UB3LYP/cc-pVTZ & -231.341 & 20.734 & 20.687 & This work \\
B3LYP/SV & -231.023 & 16.380 & 16.070 & 12 \\
CAS-MP2/6-311G(3df,2p) & & & 24.618 & 35 \\
\hline
\end{tabular}




\section{Computational details}

The search for geometries corresponding to the MECPs was done using our locally developed algorithm as described above. The SDFT calculations on the phenyl cation used B3LYP (Refs. 27 and 28) to facilitate comparison with previously published results ${ }^{12,35}$ which had used that particular exchange-correlation functional. The SDFT calculations on $\left[\mathrm{Fe}(\mathrm{ptz})_{6}\right]^{2+}$ were carried out on a geometric model based on the crystallographic structure of $\left[\mathrm{Fe}(\mathrm{ptz})_{6}\right]\left(\mathrm{BF}_{4}\right)_{2}$ (Ref. 13), as shown in the figure. All density-functional calculations on the iron complex were done using the B-PW91 $1^{31,32,36}$ exchange-correlation functional and the all-electron basis sets $6-31 \mathrm{G}^{*}$ and $6-311 \mathrm{G}^{*}{ }^{24}$ The B-PW91 functional was used because it has proven to yield fairly accurate relative electronic energies for metal-containing complexes. ${ }^{34}$ Single point density-functional calculations were done using GAUSSIAN $98 .^{37}$ Full geometry optimizations were carried out with the same package to determine the minimum-energy configurations corresponding to the singlet, triplet, and quintet states of the iron complex.

\section{RESULTS AND DISCUSSION}

\section{A. Convergence analysis of MECP algorithm}

To better understand the convergence behavior of the MECP algorithm and to further improve its convergence rate, we can analyze the formalism given by Eqs. (3)-(16). First, it is convenient to define the following vectors:

$$
\begin{aligned}
& \mathbf{p} \equiv g_{A}+g_{B}, \\
& q \equiv g_{A}-g_{B} .
\end{aligned}
$$

Second, to study how the power of the constraint equation affects the convergence rate, it is possible to generalize Eq. (4) to the $n$th power of the energy difference,

$$
C=\left(E^{M}-E^{M^{\prime}}\right)^{n}=0 .
$$

In addition, Eq. (5)-(16) can be rewritten in terms of $\mathbf{p}$ and $\mathbf{q}$ as follows:

$$
\begin{aligned}
& \mathbf{g}=\frac{\mathbf{p}}{2}, \\
& \mathbf{J}=n\left(E^{M}-E^{M^{\prime}}\right)^{n-1} \mathbf{q}, \\
& \lambda=\frac{\left(E^{M}-E^{M^{\prime}}\right)^{n}-(1 / 2) n\left(E^{M}-E^{M^{\prime}}\right)^{n-1} \mathbf{q} \cdot\left(\widetilde{H}^{-1} \mathbf{p}\right)}{n^{2}\left(E^{M}-E^{M^{\prime}}\right)^{2 n-2} \mathbf{q} \cdot\left(\widetilde{H}^{-1} \mathbf{q}\right)} \\
& =\frac{\left(E^{M}-E^{M^{\prime}}\right)-(1 / 2) n \mathbf{q} \cdot\left(\tilde{H}^{-1} \mathbf{p}\right)}{n^{2}\left(E^{M}-E^{M^{\prime}}\right)^{n-1} \mathbf{q} \cdot\left(\tilde{H}^{-1} \mathbf{q}\right)} .
\end{aligned}
$$

Hence,

$$
\lambda \mathbf{J}=\frac{\left(E^{M}-E^{M^{\prime}}\right) / n-(1 / 2) \mathbf{q} \cdot\left(\widetilde{H}^{-1} \mathbf{p}\right)}{\mathbf{q} \cdot\left(\tilde{H}^{-1} \mathbf{q}\right)} \mathbf{q} .
$$

Substituting Eqs. (30) and (34) into Eq. (15),

$$
\begin{aligned}
& d \mathbf{R}=-\widetilde{H}^{-1}\left(\frac{\mathbf{p}}{2}+\gamma \mathbf{q}\right), \\
& \gamma=\left[\frac{E^{M}-E^{M^{\prime}}}{n}-\frac{1}{2} \mathbf{q} \cdot\left(\tilde{H}^{-1} \mathbf{p}\right)\right] /\left[\mathbf{q} \cdot\left(\tilde{H}^{-1} \mathbf{q}\right)\right] .
\end{aligned}
$$

Mathematically, the previous expression is equivalent to using Eqs. (16) and (18). However, (36) is more convenient because its form does not lead to a singularity since the denominator of $\gamma$ does not vanish when $E^{M}=E^{M^{\prime}}$. Writing the stepping vector $d \mathbf{R}$ in this fashion also shows an explicit dependence on the power of the constraint equation.

To study the convergence behavior, we consider the illustrative example of two quadratic energy surfaces, as shown in Fig. 5. The surfaces are defined by Eqs. (37) and (38) and depend on a single nuclear coordinate $R$ (e.g., a bond length).

$$
\begin{aligned}
& E_{A}=\frac{1}{2} k(R-b)^{2}, \\
& E_{B}=\frac{1}{2} k(R+b)^{2} .
\end{aligned}
$$

The MECP occurs at $R=0$ where $\Delta E=E_{A}-E_{B}=0$. However, during the course of the iterative procedure, $\Delta E$ remains finite. According to Eqs. (37) and (38), at the $i$ th iteration

$$
\Delta E_{i}=-2 k b R_{i}
$$

where $R_{i}$ is the value of the nuclear coordinate at the current $i$ th iteration. To see how $\Delta E$ changes from one iteration to the next, we apply Eqs. (35) and (36) to compute $R_{i+1}$. Because, in this particular example there is only one degree of freedom, it is convenient to drop the vector notation. According to Eqs. (10), (27), and (28)

$$
\begin{aligned}
& H^{-1}=\frac{1}{k}, \\
& p=+2 k R_{i}, \\
& q=-2 k b .
\end{aligned}
$$

Hence Eqs. (35) and (36) imply that

$$
\begin{aligned}
\gamma=\left[\frac{-2 k b R_{i}}{n}-\frac{1}{2}(-2 k b) \frac{1}{k}\left(2 k R_{i}\right)\right] /\left[(-2 k b) \frac{1}{k}(-2 k b)\right], \\
=\left(1-\frac{1}{n}\right) \frac{R}{2 b} \\
d R=-\frac{1}{k}\left[\frac{1}{2}\left(2 k R_{i}\right)+\gamma(-2 k b)\right]=-\frac{R_{i}}{n}
\end{aligned}
$$

Therefore, in the next iteration,

$$
R_{i+1}=R_{i}+d R=R_{i}\left(1-\frac{1}{n}\right),
$$




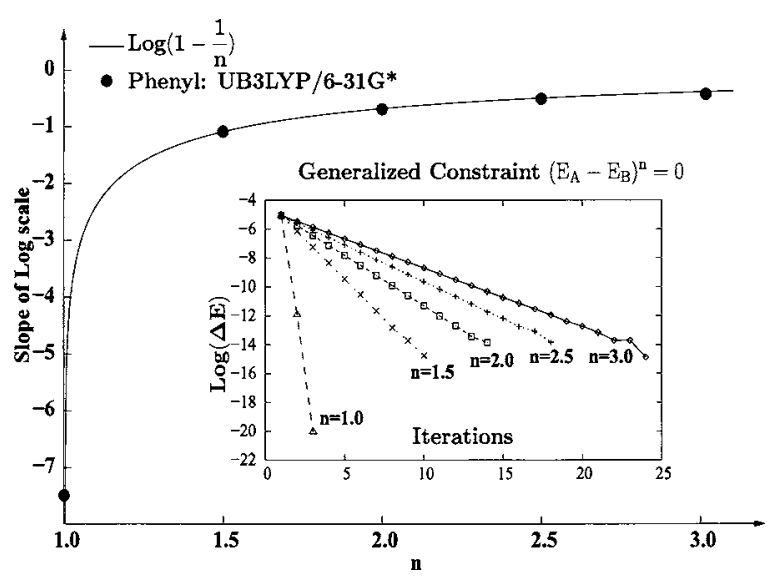

FIG. 4. Embedded box: logarithm of energy difference $\Delta E$ as a function of increasing number of iterations for various values of the constraint power $n$. The slopes reflect the convergence rate. Outer box: the slope of $\log (\Delta E)$ obtained from the calculations as a function of constraint power $n$. The dots are the values obtained for the phenyl cation using the MECP algorithm described in this work with energies obtained from the SDFT calculations at the UB3LYP/6-31G* level of theory. The solid line corresponds to the analytical expression describing the convergence rate $\log (1-1 / n)$. There is excellent agreement between computed and analytical values.

$$
\Delta E_{i+1}=\Delta E_{i}\left(1-\frac{1}{n}\right) .
$$

Equation (47) provides significant insight about the convergence behavior of our method towards the MECP. Namely, $\Delta E$ vanishes exponentially as the number of iterations increases. The convergence rate is directly related to $n$, the power of the constrain in Eq. (29),

$$
\Delta E_{i}=\Delta E_{0}\left(1-\frac{1}{n}\right)^{i}
$$

The previous derivation suggests that the fastest convergence is achieved for $n=1$. For higher values of $n$ the convergence is slower. Plotting $\Delta E$ on a logarithmic scale as a function of iteration, the slope of such graph should be $\log (1-1 / n)$. To verify that the convergence rate is indeed described by Eq. (48), we computed the MECP between singlet and triplet states of the phenyl cation using Eqs. (35) and (36) for various values of $n$. Figure 4 illustrates how the slope of the computed $\Delta E$ is in excellent agreement with the slope given by the analytical expression $\log (1-1 / n)$. In addition, the embedded box (Fig. 4) shows how $\Delta E$ decreased with increasing number of iterations. The fast convergence rate of $\Delta E$ towards zero is reflected by the slope of the graph on a logarithmic scale which shows the rate varying as a function of $n$ (Fig. 4). Furthermore, the fast convergenge of the algorithm applies not only to the crossing of two surfaces with slopes of opposite sign (i.e., $\mathbf{g}_{A}=-k \mathbf{g}_{B}, k$ being one or some other positive number), as illustrated by Fig. 5(a), but also to crossing surfaces with slopes of the same sign (i.e., $\mathbf{g}_{A}$ $\left.=k \mathbf{g}_{B}\right)$. The latter case is exemplified by Eqs. (49) and (50) and Fig. 5(b),

$$
E_{A}=\frac{1}{2} k(R-b)^{2}+a,
$$

a)

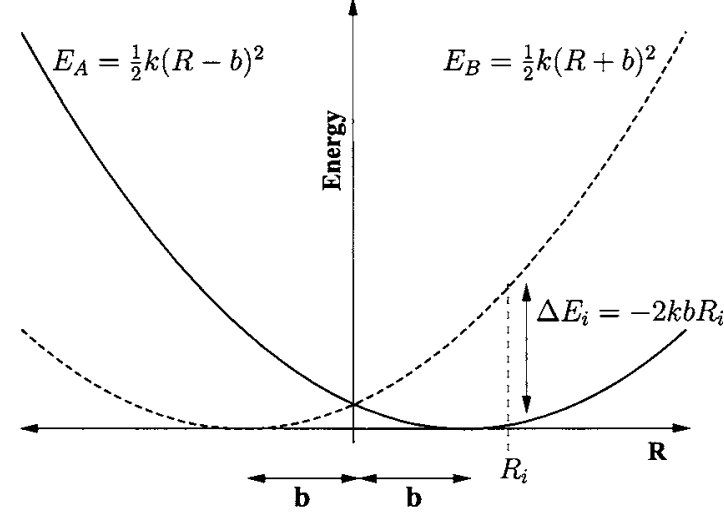

b)

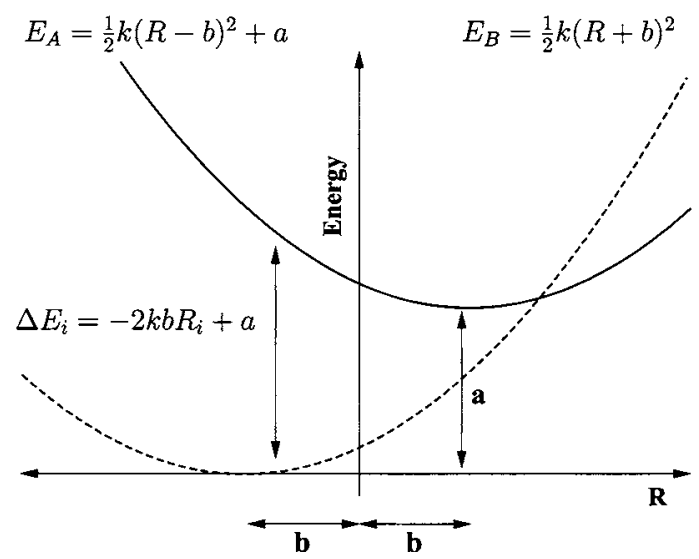

FIG. 5. Two quadratic energy surfaces which are functions of a single coordinate $R$. (a) The MECP occurs at $R=0$ where the slopes of the crossing surfaces have opposite sign. (b) The MECP occurs at a higher energy where the crossing surfaces have slopes of the same sign.

$$
E_{B}=\frac{1}{2} k(R+b)^{2}
$$

where $a>0$ shifts the energy of $E_{A}$. An extension of the previous analysis yields

$$
\begin{aligned}
& R_{i+1}=R_{i}\left(1-\frac{1}{n}\right)+\frac{a}{2 n k b}, \\
& \Delta E_{i+1}=\Delta E_{i}\left(1-\frac{1}{n}\right) .
\end{aligned}
$$

Thus, by comparison of (52) with (47) it follows that the fast logarithmic convergence is also expected for crossing slopes of the same sign. In the most general case, however, the convergence rate may depart somewhat from the previous expression to the extent that the computed crossing surfaces may not be strictly quadratic.

\section{B. Spin-forbidden intersystem crossing of phenyl cation}

To test our algorithm we have initially chosen a fairly small system that was previously studied by other authors, namely, the phenyl cation, $\left[\mathrm{C}_{6} \mathrm{H}_{5}\right]^{+}$, shown in Fig. 2. This allows us to compare our own (i) computational accuracy and (ii) algorithmic efficiency with those of other available 
methods. In fact, phenyl cation potential-energy surfaces and MECPs have been previously studied by density-functional theory and other $a b$ initio methods (MP2, CASSCF). ${ }^{12,35} \mathrm{We}$ stress, however, that although MP2 and CASSCF are practical options for small systems, these are computationally prohibitive for large metal-containing inorganic and bioinorganic complexes. Therefore, for the investigation of coordination compounds, SDFT-based methods are currently the best option for capturing correlation effects and numerical accuracy at a moderate computational cost. ${ }^{33,34}$ Our results for $\left[\mathrm{C}_{6} \mathrm{H}_{5}\right]^{+}$show that our energy-gradient SDFTbased MECP algorithm is significantly more efficient (Figs. 4 and 2) and also of comparable accuracy (Tables I and II) than other existing methods or computational implementations. ${ }^{12,35}$ For this test case our algorithm, as described in Sec. II, converged in 13 iterations for $n=2$ as compared to some 30 iterations needed for the alternative method (Fig. 2). Even faster convergence was achieved for $n=1$ and $n=1.5$, as shown in Fig. 4. This fast-converging methodology has the potential to find MECPs of more complex processes such as transitions arising in spin-forbidden radiationless decay of metallic complexes (vide infra) or in nonadiabatic spin-forbidden ligand-binding reactions of metalloproteins.

Although the ground state of the phenyl cation is known to be a singlet, it is believed that under some experimental conditions a transient triplet state is initially formed. ${ }^{12}$ This unstable spin state eventually decays into the singlet ground state. Such decay or intersystem crossing is spin forbidden because the total spin of the system is not conserved. As previously mentioned, to understand the transition from triplet to singlet states, it is important to determine the minimum-energy crossing point. ${ }^{12}$ Tables I and II show that MECP and related geometries evaluated by our algorithm are in good agreement with those of other methods used in conical intersection analysis which validates the accuracy of our

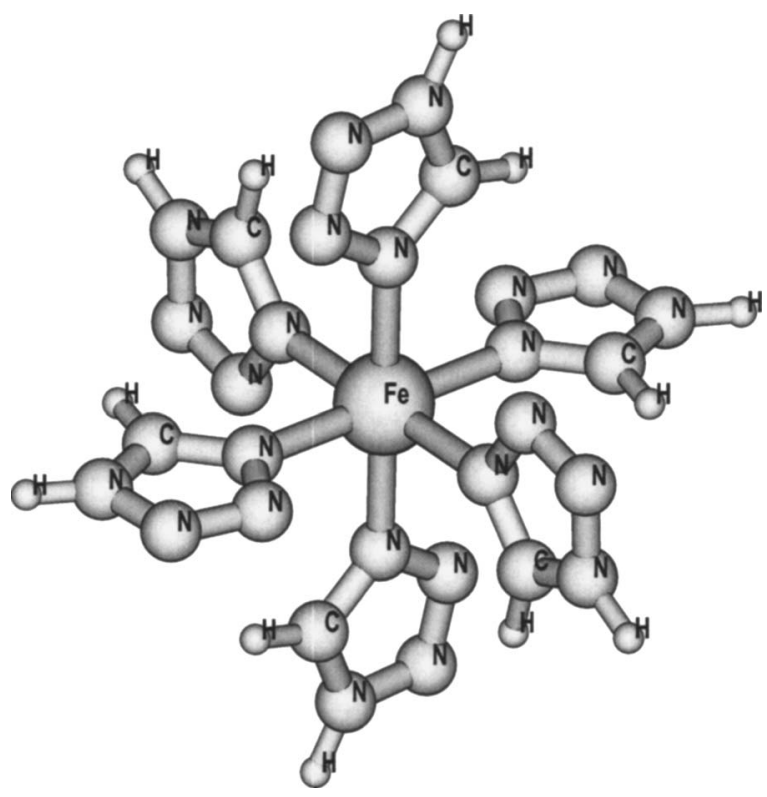

FIG. 6. Simplified crystallographic structure of $\left[\mathrm{Fe}(\mathrm{ptz})_{6}\right]^{2+}($ Ref. 13) cation used as model for the SDFT and MECP calculations. CI symmetry was assumed throughout the calculations.

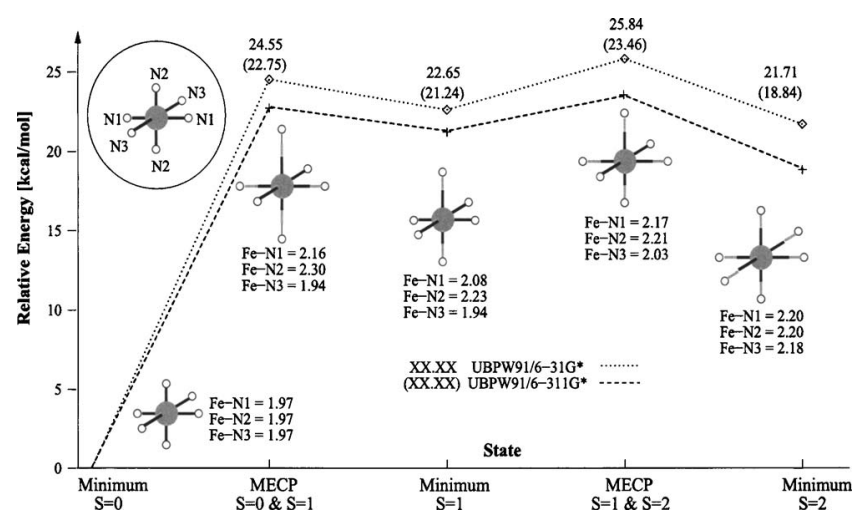

FIG. 7. Energies and geometric parameters of LIESST-exhibiting complex $\left[\mathrm{Fe}(\mathrm{ptz})_{6}\right]^{2+}$ computed for the minimum-energy configurations corresponding to ground-state singlet, intermediate triplet state, and metastable quintet state. The corresponding energies and geometric parameters for the computed MECPs are also shown. The bond lengths are given in Angstrom. The energies are given for two different basis sets $\left(6-31 \mathrm{G}^{*}\right.$ and $\left.6-311 \mathrm{G}^{*}\right)$ which yield similar trends.

method. More importantly, our algorithm is not only of comparable accuracy but also significantly more efficient.

We note that treating both spin energy surfaces on equal footing, via Eq. (3), significantly increased the efficiency of our algorithm. For our initial guess of geometric parameters [Fig. 2(a)] the energy difference between the singlet and triplet states was on the order of 0.1 hartree $\left(10^{2} \mathrm{kcal} / \mathrm{mol}\right)$. As shown in Fig. 2, with subsequent iterations the solid lines representing the convergence of the two energy surfaces were well behaved (i.e., the nuclear coordinates converged smoothly towards the MECP geometry). By contrast, the convergence of the alternative method (dashed lines) showed an oscillatory behavior for the singlet energy surface whereas the triplet surface was well behaved throughout the iteration cycle. These different convergence behaviors likely result from the fact that the latter method treats the singlet and triplet surfaces differently as evidenced by its definition of gradient given by Eq. (23).

\section{Application to spin crossover in iron coordination compounds}

Recently, the notion of photoswitchable compounds has become a growing area of research whereby it is conceivable to exploit spin degrees of freedom of molecular systems to store bits of information. ${ }^{15}$ In particular, the iron-containing complex $\left[\mathrm{Fe}(\mathrm{ptz})_{6}\right]\left(\mathrm{BF}_{4}\right)_{2}(\mathrm{ptz}=1$-propyltetrazole) (Ref. 14) can change its total spin upon optical excitation and is subsequently trapped in a new metastable spin state. Such phenomena is named light-induced excited spin state trapping (LIESST). $\left[\mathrm{Fe}(\mathrm{ptz})_{6}\right]\left(\mathrm{BF}_{4}\right)_{2}$ is a distorted octahedral $\mathrm{Fe}(\mathrm{II})$ complex (Fig. 6) with singlet ground state $(S=0)$ and exhibits a deeply red color in crystalline form. ${ }^{13,38}$ Upon irradiation by green light, the crystal becomes colorless and is trapped in a high spin excited state $(S=2)$. Remarkably, the complex remains in that metastable quintet state as long (order of days) as the temperature is kept under $50 \mathrm{~K}^{14}$ At higher temperatures, however, the lifetime of the metastable high spin state decreases (e.g., on the order of minutes at $60 \mathrm{~K})$. 


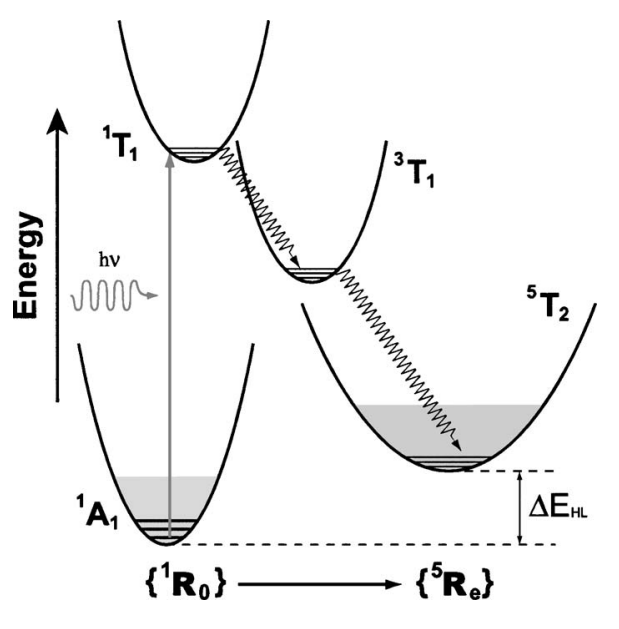

SCHEME 1.

Some progress has been made in understanding the mechanism of LIESST, mostly based on spectroscopy. ${ }^{15,16}$ However, further theoretical understanding is needed about the magnetostructural correlations and detailed electronic structures of LIESST-exhibiting complexes. Such understanding will likely lead to the synthesis of LIESST complexes with higher critical temperatures (below which the high spin state is effectively trapped) for use in potentially novel memory-storage or display applications. ${ }^{15,16}$

The basic mechanism for light-induced high spin trapping in $\left[\mathrm{Fe}(\mathrm{ptz})_{6}\right]\left(\mathrm{BF}_{4}\right)_{2}$ has been described as a dipole allowed excitation from the singlet ground state to another short-lived, higher-energy, singlet (Scheme 1)1. This is followed by spin-forbidden intersystem crossing to an intermediate lower-energy triplet which, being mediated by SOC, changes the total spin of the system by one unit. Another spin-forbidden intersystem crossing brings the system to the metastable quintet state where it is subsequently trapped below its critical temperature. ${ }^{14-16}$ Scheme 1 illustrates the mechanism of excitation by green light whereby the solid arrow $\left({ }^{1} A_{1} \rightarrow{ }^{1} T_{1}\right)$ represents the spin allowed vertical transition and the wavy arrows $\left({ }^{1} T_{1} \rightarrow{ }^{3} T_{1},{ }^{3} T_{1} \rightarrow{ }^{5} T_{2}\right)$ represent spin-forbidden radiationless intersystem crossing. It is also known that upon optical excitation the metastable quintet can relax back to the singlet ground state (reverse-LIESST) following a different excitation-deexcitation pathway. ${ }^{14-16}$ However, some fundamental questions remain unresolved. In particular, (i) which are the detailed geometries at the MECPs where the spin crossovers are most likely to take place? and (ii) how are the total electronic energies of the various spin states and MECPs involved in the LS $\leftrightarrow \mathrm{HS}$ transition related to each other? In particular, what is the energy difference between the low spin ground state and the high spin metastable state? In this work, we have applied the newly developed algorithm to elucidate the geometries and energies of $\left[\mathrm{Fe}(\mathrm{ptz})_{6}\right]^{2+}$ at these key points along the LIESST pathway.

The geometric model used in the calculations was based on the x-ray crystallographic structure of $\left[\mathrm{Fe}(\mathrm{ptz})_{6}\right]\left(\mathrm{BF}_{4}\right)_{2}$ (Ref. 13) and is shown in Fig. 6 as a cation of charge 2+. The $\mathrm{Fe}(\mathrm{II}) d^{6}$ ion can take three different spin multiplicities: singlet, triplet, and quintet. As a result, there are two possible
TABLE III. Energies of $\left[\mathrm{Fe}(\mathrm{ptz})_{6}\right]^{2+}[\mathrm{kcal} / \mathrm{mol}]$ relative to the singlet ground state evaluated at minima of various spin multiplicities and at MECPs.

\begin{tabular}{lcc}
\hline \hline & UBPW91/6-31G* & UBPW91/6-311G \\
\hline${ }^{1} \Psi$ Minimum & 0 & 0 \\
$E\left(\mathrm{MECP}^{1 \leftrightarrow 3}\right)$ & 24.546 & 22.753 \\
${ }^{3} \Psi$ Minimum & 22.646 & 21.239 \\
$E\left(\mathrm{MECP}^{3 \leftrightarrow 5}\right)$ & 25.841 & 23.456 \\
${ }^{5} \Psi$ Minimum & 21.714 & 18.841 \\
\hline \hline
\end{tabular}

crossing points (MECPs) that can be mediated by spin-orbit coupling, namely, $\mathrm{MECP}^{1 \leftrightarrow 3}$ and $\mathrm{MECP}^{3 \leftrightarrow 5}$. Once the MECPs were found, SDFT calculations were performed on all five relevant geometries (i.e., corresponding to ${ }^{1,3,5} \Psi$ minima and two MECPs). The minimum-energy geometries corresponding to each spin state $\left({ }^{1,3,5} \Psi\right)$ were obtained from full geometry optimizations whereas those at the crossing points were obtained using the MECP algorithm previously described. To test the convergence behavior of the MECP algorithm for this iron complex, $\mathrm{MECP}^{1 \leftrightarrow 3}$ was computed for $n=1$ and also for $n=2$ in the constraint equation (4). The former required 5 iterations for convergence whereas the latter required 16, in agreement with the trend predicted by Eq. (47) but also reflecting the fact that these surfaces are not strictly quadratic. The relevant energies are shown in Table III.

One of the most striking features of $\left[\mathrm{Fe}(\mathrm{ptz})_{6}\right]\left(\mathrm{BF}_{4}\right)_{2}$ is that, upon cooling, its transition from metastable high spin (HS: $S=2$ ) to low spin (LS:S=0) states is accompanied by a dramatic structural rearrangement whereby the Fe-ligand bond lengths decrease by $\approx 0.2 \AA$. In fact, at the $\mathrm{HS} \rightarrow \mathrm{LS}$ crossover temperature of $\approx 130 \mathrm{~K}$, a rhombohedral $\rightarrow$ triclinic structural phase transition has been observed. ${ }^{13,38}$ Furthermore, powder studies on the isostructural and closely related complex $\left[\mathrm{Fe}(\mathrm{ptz})_{6}\right]\left(\mathrm{ClO}_{4}\right)_{2}$ indicated that its structure had already changed before the spin transition began. ${ }^{13}$ Thus, to explain these experimental observations it is of interest to gain theoretical insight about any structural changes that may occur during the overall spin transition pathway, $S=2 \leftrightarrow S$ $=1 \leftrightarrow S=0$, since detailed structural information is presently limited to only the $S=2\left({ }^{5} \Psi\right)$ and $S=0\left({ }^{1} \Psi\right)$ states. Our calculations provide significant insight about the structural and energetic variations that occur along the overall transition pathway. In particular, at the intermediate $S=1$ state $\left({ }^{3} \Psi\right)$ and at MECPs where the $\Delta S=1$ transitions are most likely to take place.

Figure 7 displays the energies and geometric details at each stage of the LIESST pathway. The computed singlet ground-state geometry showed good agreement with the $\mathrm{X}$-ray crystallographic structure ${ }^{13}$ and also with previous theoretical studies. ${ }^{39}$ The likelihood for the system to make transitions from singlet to triplet and from triplet to quintet states is directly related to the strength of SOC at their respective MECPs. Thus, to arrive at the minimum of the intermediate triplet $\left({ }^{3} \Psi\right)$, which is $\approx 22 \mathrm{kcal} / \mathrm{mol}$ higher in energy than the singlet (Table III), the system should pass with high probability through their minimum-energy crossing point where the mediating SOC is expected to be strongest. We 
find that at the singlet-triplet crossing point the axial ligands elongated to their largest bond lengths in comparison with any other state $(\mathrm{Fe}-\mathrm{N} 2: 2.30 \AA)$. By contrast, once the system has made a transition to the intermediate triplet state, its geometry is characterized by a mixture of singlet and quintet parameters. Indeed, the axial ligands exhibited long bond lengths similar to those of the quintet whereas those in the plane retained the short bond-length characteristic of the singlet. To reach the metastable quintet state the system should pass with high probability through the crossing between the triplet and quintet states. At this MECP the geometry was closer, but not identical, to that of the quintet since most ligands displayed long bond lengths. However, two of the six ligands displayed short bond lengths at $\mathrm{MECP}^{3 \leftrightarrow 5}$. These results are consistent with the powder studies on the isostructural diperchlorate complex ${ }^{13}$ which indicate structuralchanges prior to the actual spin transition. Finally, as the system reaches the metastable quintet state its various $\mathrm{Fe}-\mathrm{N}$ bond lengths are $0.21 \leftrightarrow 0.23 \AA$ longer than the singlet, in fairly good agreement with experiment. ${ }^{13}$

\section{CONCLUSIONS}

An efficient method for locating minimum-energy crossing points has been introduced and tested against an alternative method for a well-known spin-crossover system, namely, the phenyl cation. The quantitative parameters (e.g., geometries and energies of MECPs) obtained with the present method are in good agreement with those obtained by alternative methods. However, the convergence rate of the present algorithm is significantly faster than our implementation of an alternative method and obeys a fast logarithmic law which has been verified for the phenyl cation. Due to its rapid convergence, the present method is particularly suitable for studying spin crossover in large molecular systems and, in particular, in transition metal-containing complexes. More specifically, our method significantly reduces the CPU time needed for convergence (relative to the alternative method) in two ways: (i) the number of iteration steps needed to reach the MECP is significantly lower and (ii) the number of selfconsistent field (SCF) cycles used in the SDFT evaluations of $E^{M}$ and $E^{M^{\prime}}$ at each iteration is generally lower since SCF wave functions of previous iterations are better initial guesses for subsequent iterations.

As an application of this new methodology, the minimum-energy crossing points of the technologically relevant $\left[\mathrm{Fe}(\mathrm{ptz})_{6}\right]^{2+}$ cation have been studied to identify the geometrical parameters associated with its $S=0 \leftrightarrow S=1$ and $S=1 \leftrightarrow S=2$ transitions. Our calculations show that the transition from the singlet ground state to a triplet intermediate state is accompanied by an unusually large $(\approx 0.3 \AA)$ bondlength elongation of the axial ligands. In addition, detailed energetic and structural information have been obtained for other points along the LS $\leftrightarrow$ HS pathway which are consistent with experimental information when available.

\section{ACKNOWLEDGMENTS}

This research has been partially funded by NSF Grant No. CHE-0349189 (J.H.R.) and the Purdue University Research Foundation. Generous computer time facilitated by Purdue's ITaP supercomputer center and the National Center for Supercomputer Applications (NCSA) is appreciated. We thank Taisuke Minagawa and Zhi Qi for assistance preparing the manuscript.

${ }^{1}$ M. Bearpark, M. Robb, and H. Schlegel, Chem. Phys. Lett. 223, 269 (1994).

${ }^{2}$ M. A. Robb, M. Garavelli, M. Olivucci, and F. Bernardi, Rev. Comput. Chem. 15, 87 (2000).

${ }^{3}$ R. Poli and J. Harvey, Chem. Soc. Rev. 32, 1 (2003).

${ }^{4}$ T. J. Martinez, Chem. Phys. Lett. 272, 139 (1997).

${ }^{5}$ D. R. Yarkony, J. Am. Chem. Soc. 114, 5406 (1992).

${ }^{6}$ D. R. Yarkony, J. Phys. Chem. 97, 4407 (1993).

${ }^{7}$ N. Koga and K. Morokuma, Chem. Phys. Lett. 119, 371 (1985).

${ }^{8}$ D. R. Yarkony, J. Chem. Phys. 92, 2457 (1990).

${ }^{9}$ M. R. Manaa and D. R. Yarkony, J. Chem. Phys. 99, 5251 (1993).

${ }^{10}$ I. V. Khavrutskii, D. G. Musaev, K. Morokuma, J. Am. Chem. Soc. 125, 13879 (2003).

${ }^{11}$ M. Lundberg and E. M. Siegbahn, Chem. Phys. Lett. 401, 347 (2005).

${ }^{12}$ J. Harvey, M. Aschi, H. Schwarz, and W. Koch, Theor. Chem. Acc. 99, 95 (1998).

${ }^{13}$ L. Wiehl, Acta Crystallogr., Sect. B: Struct. Sci. 49, 289 (1993).

${ }^{14}$ S. Decurtins, P. Gutlich, K. M. Hasselbach, A. Hauser, and H. Spiering, Inorg. Chem. 24, 2174 (1985).

${ }^{15}$ P. Gutlich, Y. Garcia, and T. Woike, Coord. Chem. Rev. 219, 839 (2001).

${ }^{16}$ A. Hauser, A. Vef, and P. Adler, J. Chem. Phys. 95, 8710 (1991).

${ }^{17}$ W. Kohn and L. J. Sham, Phys. Rev. 140, A1133 (1965).

${ }^{18}$ R. G. Parr and W. Yang, Density-Functional Theory of Atoms and Molecules (Clarendon Press, Oxford, 1989).

${ }^{19}$ W. Kohn, A. D. Becke, and R. G. Parr, J. Phys. Chem. 100, 12974 (1996).

${ }^{20} \mathrm{R}$. Bock and W. Chrischer, The Data Analysis BriefBook (CERN, Geneva, 1998).

${ }^{21}$ J. Simons, P. Jorgensen, H. Taylor, and J. Ozment, J. Phys. Chem. 87, 2745 (1983).

${ }^{22}$ H. B. Schlegel, J. Comput. Chem. 3, 214 (1982).

${ }^{23}$ W. H. Miller, N. C. Handy, and J. E. Adams, J. Chem. Phys. 72, 99 (1980).

${ }^{24}$ W. J. Hehre, L. Radom, P. v.R. Schleyer, and J. A. Pople, Ab Initio Molecular Orbital Theory (Wiley, New York, 1986).

${ }^{25}$ P. E. M. Siegbahn, in New Methods in Computational Quantum Mechanics, edited by I. Prigogine and S. A. Rice (Wiley, New York, 1996).

${ }^{26}$ M. Head-Gordon, J. Phys. Chem. 100, 13213 (1996).

${ }^{27}$ A. D. Becke, J. Chem. Phys. 98, 5648 (1993).

${ }^{28}$ A. D. Becke, J. Chem. Phys. 98, 1372 (1993).

${ }^{29}$ C. Lee, W. Yang, and R. G. Parr, Phys. Rev. B 37, 785 (1988).

${ }^{30}$ A. D. Becke, Phys. Rev. A 38, 3098 (1988).

${ }^{31}$ J. P. Perdew, Phys. Rev. B 33, 8822 (1986).

${ }^{32}$ J. P. Perdew and Y. Wang, Phys. Rev. B 45, 13244 (1992).

${ }^{33}$ J. H. Rodriguez, D. E. Wheeler, and J. K. McCusker, J. Am. Chem. Soc. 120, 12051 (1998).

${ }^{34}$ J. H. Rodriguez and J. K. McCusker, J. Chem. Phys. 116, 6253 (2002).

${ }^{35}$ A. Nicolaides, D. Smith, F. Jensen, and L. Radom, J. Am. Chem. Soc. 119, 8083 (1997).

${ }^{36}$ A. D. Becke, Phys. Rev. A 38, 3098 (1998)

${ }^{37}$ M. J. Frisch, G. W. Trucks, H. B. Schlegel et al., GAUSSIAN 98, Revision A.4, Gaussian, Inc., Pittsburgh, PA, 1998.

${ }^{38}$ L. Wiehl, H. Spiering, P. Gütlich, and K. Knorr, J. Appl. Crystallogr. 23, 151 (1990).

${ }^{39}$ M. Kondo and K. Yoshizawa, Chem. Phys. Lett. 372, 519 (2003).

${ }^{40}$ See EPAPS Document No. E-JCPSA6-123-723530 for a figure showing the flow diagram of the MECP algorithm and five tables with optimized and MECP Cartesian coordinates of $\left[\mathrm{Fe}(\mathrm{ptz})_{6}\right]^{2+}$ at the UBPW91/6 $-311 \mathrm{G}^{*}$ level. This document can be reached via a direct link in the online article's HTML reference section or via the EPAPS homepage (http:// www.aip.org/pubservs/epaps.html). 